\title{
Cerebral tumefactive demyelinating lesions
}

\author{
WEI QI, GE JIA, XINSHENG WANG, MAOZHI ZHANG and ZHENYU MA
}

Department of Neurosurgery, Beijing Tiantan Hospital Affiliated to Capital Medical University, Beijing 100050, P.R. China

Received August 13, 2014; Accepted April 24, 2015

DOI: $10.3892 / 01.2015 .3481$

\begin{abstract}
Tumefactive demyelinating lesions (TDLs), are a rare demyelinating pathological disease in the central neurological system, which have been proven to be a diagnostic dilemma to neurosurgeons. The clinical presentation and radiographic appearance of these lesions often results in their misdiagnosis as intracranial tumors, such as gliomas, which leads to unnecessary surgical resection and adjunct radiation. In the present study, the clinical and radiographic features of 14 patients with cerebral TDLs who underwent surgical treatment between January 2004 and January 2009 were reviewed and analyzed. The surgical methods used included biopsy and resection, while steroid therapy was indicated when TDLs were confirmed by histopathological analysis. The patients were followed-up and the outcomes were evaluated using the Karnofsky performance scale (KPS). The main clinical presentations included: Hemiplegia ( 8 cases), increased intracranial pressure (4 cases) and seizures (general in 1 case; partial in 3 cases). On magnetic resonance imaging scans, 12/14 TDL cases demonstrated an isolated local subcortical mass and 6/14 cases (42.9\%) demonstrated enhancing veins coursing undistorted through the lesion. The postoperative complications included: Hemiplegia (2 cases) and mortality (1 case). A total of 9 cases underwent microsurgical total resection, and 5 cases received stereotactic biopsy that was followed with high-dose methylprednisolone therapy. The follow-up study demonstrated that 2 cases presented recurrence with multiple sclerosis and the KPS scores for $13 / 14$ patients $(92.9 \%)$ were $\geq 80$. In conclusion, the clinical and radiographic features of TDLs may help to establish the correct diagnosis prior to surgery, in order to avoid unnecessary resection or adjunctive therapy. Using steroid therapy, the majority of patients with TDLs appeared to achieve satisfactory prognosis.
\end{abstract}

Correspondence to: Dr Zhenyu Ma, Department of Neurosurgery, Beijing Tiantan Hospital Affiliated to Capital Medical University, 6 Tiantan Xili, Chongwen, Beijing 100050, P.R. China

E-mail: zhenyuma06@sina.com

Key words: tumefactive demyelinating lesions, clinical features, steroid therapy

\section{Introduction}

Demyelinating diseases, are neuropathological entities most frequently observed in the central nervous system with a preferential involvement of the major white matter tracts in a periventricular distribution. These diseases are often difficult to diagnose, since they do not exhibit a typical appearance on magnetic resonance imaging (MRI) scans (1-3). Tumefactive demyelinating lesions (TDLs), also referred to as 'demyelinating pseudo tumors', are a rare type of demyelinating disease. They manifest as solitary lesions or a few separate lesions that result in a mass effect, edema on the surrounding tissue and enhancement $(4,5)$. The correct diagnosis is often not established by radiological study, but only after surgical biopsy or resection (6-8). TDLs were first reported by van der Velden et al (9) in 1979. Pathologically, TDLs are considered to be intermediate lesions between multiple sclerosis and acute disseminated encephalomyelitis (8). The clinical presentation and radiographic appearance of these lesions often result in the diagnosis of intracranial tumors, including gliomas $(8,10-12,13)$. Since the majority of patients with TDLs respond favorably to corticosteroid treatment, surgical resection is not required; therefore, it is important for neurosurgeons to improve their understanding of this uncommon pathological process in order to avoid unnecessary resection or adjunctive therapy. The present study analyzed the data of 14 patients with TDLs who underwent surgical treatment in the Department of Neurosurgery, Beijing Tiantan Hospital (Beijing, China) between January 2004 and January 2009 to help to establish the correct diagnosis prior to surgery, in order to avoid unnecessary resection or adjunctive therapy in future cases of TDLs.

\section{Materials and methods}

Patients. Between January 2004 and January 2009, 14 patients (male, 9; female, 5) with cerebral TDLs were admitted and received surgical treatment at the Department of Neurosurgery of the Beijing Tiantan Hospital. The median age was 24 years (range, 4-51 years). The diagnosis of TDLs was established following pathological analysis. All the patients were examined neurologically and radiologically, including the use of computed tomography (CT) and MRI, prior to and following surgery. The clinical presentations, imaging features and postoperative neurological deficits were recorded. The study was approved by the Ethics Committee of Beijing Tiantan Hospital Affiliated to Capital Medical University 
(Beijing, China). Written informed consent was obtained from the patients/patients' families.

Treatment. The surgical approach was decided according to the preoperative diagnosis, which was performed based on the result of medical history, presentations and the radiology examination (CT and MRI scans). When the preoperative diagnosis was considered to be a tumor, craniotomy and microsurgical resection were performed with the aim of complete or partial resection depending on what was feasible. However, in cases where it was not possible to affirm a certain preoperative diagnosis, biopsy was indicated. Biopsy of suspicious lesions was approached stereo tactically in order to minimize tissue injury. In order to improve the positive rate and accuracy of the biopsy, 9-12 targets were selected during stereotactic biopsy. Immunohistopathological analysis was performed for all cases, while the frozen-section procedure was performed only in cases of surgical resection. For the cases that received biopsy, corticosteroid therapy using high-dose methylprednisolone was administered once the diagnosis of TDL was confirmed by histopathological examination. The treatment regimen was as follows: 500-1,000 mg of methylprednisolone within $500 \mathrm{ml}$ of $0.9 \% \mathrm{NaCl}$ or $5 \%$ glucose was administrated intravenously by dropping in $3 \mathrm{~h}$ per day for 5 days, which was followed by oral administration of 60-80 mg per day for 1 week. Subsequently, $10 \mathrm{mg}$ methylprednisolone was administered every 5 days to allow withdrawal. Postoperatively, patients with a history of seizure were treated continuously with anticonvulsant therapy until 1-2 years free of seizures was achieved. By contrast, for patients with no history of seizures, 3 months of prophylactic anticonvulsant therapy was routinely administered.

Follow-up. Follow-up study was conducted for all the patients by routine outpatient appointments with neurological and neuroradiological examinations at 3-6 months following discharge, and later by interview, telephone or post. The Karnofsky performance scale (KPS) was used to evaluate the patients' status (15).

Data presentation. Continuous variables were expressed as the mean \pm standard deviation or the median (range), and categorical variables were expressed as proportions.

\section{Results}

Clinical presentations. From the available data of 14 patients, the male-to-female ratio was $\sim 1.8$, with a male preponderance (Table I).

TDLs were considered to have an acute (4 cases), sub-acute (6 cases) or chronic (4 cases) onset with a duration (defined as the time between onset and admission) of 10 days to 7 years. The main symptoms and signs of the TDLs included the following: Increased intracranial pressure (ICP, 4 cases); local neurological deficits, including hemiplegia (8 cases) and partial aphasia (1 case); seizures, including general (1 case) and local (3 cases). Only 1 case had a history of vaccination and no cases were observed to have an associated infection history.

Radiographic features. The radiographic features observed in the 14 TDL cases are listed in Table II. The locations of
Table I. Clinical presentations of 14 patients with tumefactive demyelinating lesions.

\begin{tabular}{lcc}
\hline Clinical presentation & Cases, $\mathrm{n}$ & Proportion, \% \\
\hline Severity & 4 & 28.6 \\
$\quad$ Acute & 6 & 42.8 \\
Subacure & 4 & 28.6 \\
Chronic & & \\
Symptoms and sings & 4 & 28.6 \\
ICP & & \\
Local neurological deficits & 8 & 57.2 \\
Hemiplegia & 1 & 7.1 \\
Partial aphasia & & 7.1 \\
Seizures & 1 & 21.4 \\
General & 3 & 7.1 \\
Local & 1 & 0.0 \\
Vaccination history & 0 & \\
Infection history & & \\
\hline ICP, increased intracranial pressure. & & \\
\hline
\end{tabular}

Table II. Radiographic features of tumefactive demyelinating lesions patients.

\begin{tabular}{|c|c|c|}
\hline Feature & Cases, $\mathrm{n}$ & Proportion, $\%$ \\
\hline \multicolumn{3}{|l|}{ Solitary lesions } \\
\hline Frontal lobe & 3 & 21.4 \\
\hline Temporal lobe & 4 & 28.6 \\
\hline Parietal lobe & 3 & 21.4 \\
\hline Insular lobe & 1 & 7.2 \\
\hline Basilar ganglia area & 1 & 7.2 \\
\hline \multicolumn{3}{|l|}{ Multiple lesions ${ }^{\mathrm{a}}$} \\
\hline Frontal lobe + parietal lobe & 1 & 7.2 \\
\hline Frontal lobe + pons & 1 & 7.2 \\
\hline \multicolumn{3}{|l|}{ Computed tomography scan } \\
\hline Hypodense $^{\mathrm{b}}$ & 9 & 64.3 \\
\hline Heterogeneous density & 5 & 35.7 \\
\hline \multicolumn{3}{|l|}{ Magnetic resonance imaging ${ }^{\mathrm{c}}$} \\
\hline Local subcortical mass & 12 & 85.7 \\
\hline Heterogeneous intensity & 2 & 14.3 \\
\hline Relative sharp margin ${ }^{\mathrm{d}}$ & 6 & 42.9 \\
\hline Poor defined margin & 8 & 57.1 \\
\hline \multicolumn{3}{|l|}{ Variable enhancement } \\
\hline Patchy & 5 & 35.7 \\
\hline Open ring-like & 6 & 42.9 \\
\hline Ring-like & 3 & 21.4 \\
\hline Vein coursing & 6 & 42.9 \\
\hline
\end{tabular}

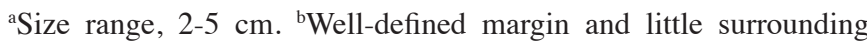
edema. 'Hypointense on T1-weighted images; hyperintense on T2-weighted and fluid-attenuated inversion recovery images. ${ }^{\mathrm{d}}$ Light to moderate surrounding edema. 
A

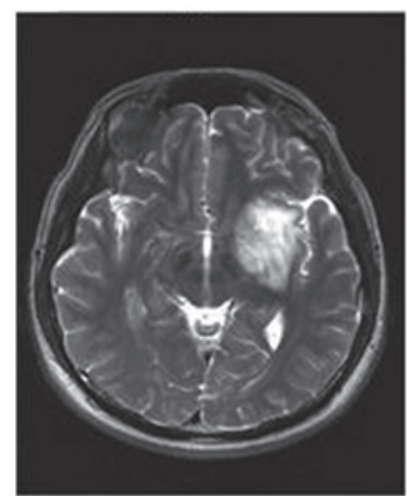

B

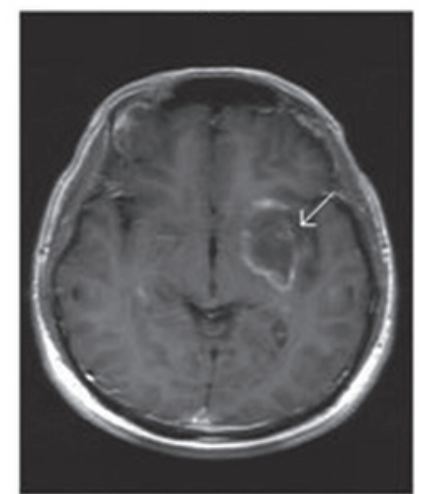

D

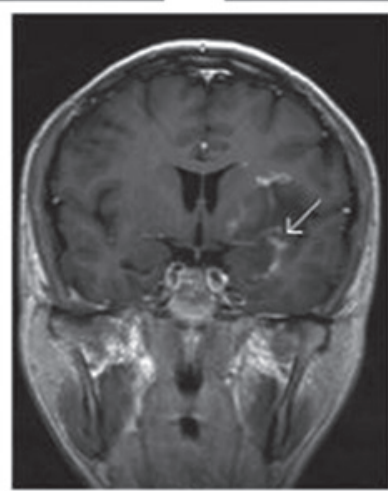

$\mathbf{E}$

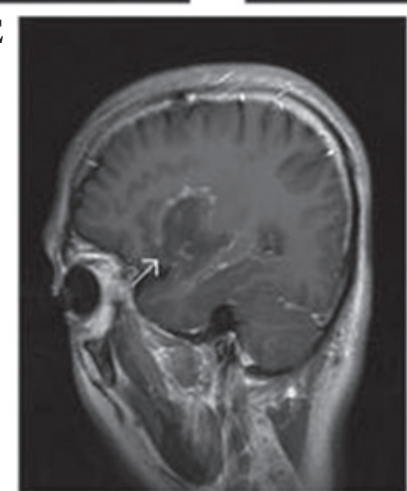

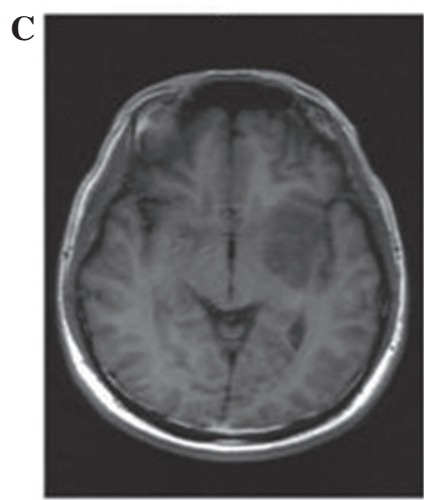

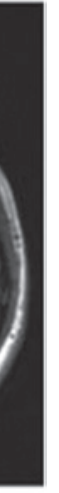

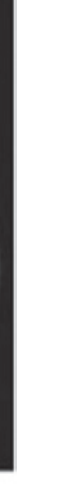

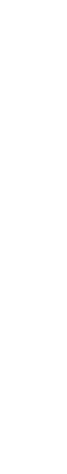

(1) within left basal ganglion area. (B) Enhanced axial T1-weighted image revealing ring-like enhancement. (C) Non-enhanced axial T1-weighted image. of the lesion (D) Enhanced coronal T1-weighted image of the lesion. (E) Enhanced coronal T1-weighted image of the lesion. The arrows indicate the area of the lesion.

Table III. Diagnosis of patients with tumefactive demyelinating lesions.

\begin{tabular}{lcc}
\hline Diagnosis & Cases, $\mathrm{n}$ & Proportion, \% \\
\hline Certain diagnosis & & \\
Gliomas & 8 & 57.1 \\
Metastasis & 1 & 7.1 \\
No certain diagnosis & 5 & 35.8 \\
\hline
\end{tabular}

Table IV. Therapy methods and outcomes of patients with tumefactive demyelinating lesions.

\begin{tabular}{lcc}
\hline Parameter & Cases, $\mathrm{n}$ & Proportion, \% \\
\hline Resection & & \\
$\quad$ Total & 8 & 57.1 \\
$\quad$ Subtotal (frozen-section) & 1 & 7.1 \\
Intracranial hypotension & 4 & 28.6 \\
Complication & & \\
$\quad$ Hemiplegia & 2 & 14.3 \\
$\quad$ Mortality & 1 & 7.1 \\
Biopsy & 5 & 35.7 \\
Symptoms and signs & & \\
$\quad$ Improved & 4 & 28.6 \\
$\quad$ Relieved & 2 & 14.3 \\
\hline
\end{tabular}

solitary lesions (12 cases) included the following: Frontal lobe (3 cases), temporal lobe ( 4 cases), parietal lobe ( 3 cases), insular lobe (1 case) and basilar ganglia area (1 case). Regarding the 2 cases with multiple lesions, in 1 case the lesions were located at the frontal lobe and parietal lobe, while in the other case the lesions were located in the frontal lobe and the pons. The size of the lesions ranged between 2 and $5 \mathrm{~cm}$. On the CT scans, the TDLs were hypodense ( 9 cases) or heterogeneously dense (5 cases) masses with a well-defined margin and little surrounding edema. On the MRI scans, 12 cases demonstrated local subcortical masses which were hypo-intense on T1-weighted images and hyper-intense on T2-weighted images and fluid-attenuated inversion recovery images, with the exception of 2 cases with heterogeneous signal intensity. A total of 6 cases had a relatively sharp margin and 8 cases had a poorly defined margin, with light to moderate surrounding edema. Following Gd-DTPA administration, all the cases presented variable enhancement, including 5 cases (35.7\%) of patchy, 6 cases of open ring-like (42.9\%) and 3 cases of ring-like (21.4\%) enhancement. In addition, 6 cases demonstrated enhancing vein coursing undistorted through the lesion (Fig. 1).

Diagnosis, treatments and outcomes. Among the 14 cases examined, 8 cases were misdiagnosed preoperatively as gliomas and 1 case as metastasis (Table III). In 5 cases, no certain diagnosis was established. Microsurgical resection was performed in 9 cases, while 5 cases were approached by stereotactic biopsy. Gross complete resection was achieved in 8 patients and partial resection in 1 patient, which was suspicious of TDL 
Table V. Follow-up study.

\begin{tabular}{|c|c|c|}
\hline Outcome & Cases, n (\%) & Notes \\
\hline \multicolumn{3}{|l|}{ Complications } \\
\hline Hemiplegia & $4(28.6)$ & $\begin{array}{l}2 \text { resection cases; } \\
2 \text { biopsy cases }\end{array}$ \\
\hline Seizures & $4(28.6)$ & $\begin{array}{l}2 \text { cases improved; } \\
2 \text { cases achieved total } \\
\text { seizure-control }\end{array}$ \\
\hline Aphasia & 1 & $\begin{array}{l}\text { Totally relieved } 3 \text { months } \\
\text { after biopsy }\end{array}$ \\
\hline Recurrent cases & $2(14.3)$ & $\begin{array}{l}\text { Identified by MRI, } 26 \text { and } \\
51 \text { months postoperatively }\end{array}$ \\
\hline \multicolumn{3}{|l|}{ KPS } \\
\hline 100 & $7(50.0)$ & - \\
\hline 90 & $3(21.4)$ & - \\
\hline 80 & $3(21.4)$ & - \\
\hline 0 & $1(7.1)$ & Mortality \\
\hline
\end{tabular}

MRI, magnetic resonance imaging; KPS, Karnofsky performance scale.

by frozen-section (Table IV). For the 9 patients that underwent resection, the ICP was relieved completely in 4 patients following surgery. The postoperative complications included hemiplegia in 2 cases and mortality in 1 case (due to postoperative intracranial infection). For all the biopsy cases, there were no surgical complications in the present study. All the biopsy cases received high-dose methylprednisolone therapy after the diagnosis of TDLs was confirmed by histopathological examination. Following steroid therapy, the preoperative symptoms and signs improved (4 cases) or totally relieved ( 2 cases) for all 6 patients (improved and relieved) in 1-4 weeks (Table IV).

Follow-up study. The follow-up study (range, 17-72 months; mean, 41 months) was successfully completed in all 14 cases, with the exception of 1 mortality case. Among them, 2 resection cases and 2 biopsy cases still had hemiplegia, which was improved compared with that at discharge (Table V). A total of 2 cases with seizures were improved and the other 2 cases achieved total seizure-control. The partial aphasia was completely relieved in 3 months following biopsy. There were 2 recurrence cases $(14.3 \%)$ identified by MRI, at 26 and 51 months postoperatively, which presented as multiple sclerosis and were improved by steroid therapy. The KPS assessment demonstrated a score of 100 for 7 cases $(50 \%), 90$ for 3 cases (21.4\%), 80 for 3 cases (21.4\%), and 0 for 1 case (7.1\%) (16).

\section{Discussion}

The exact pathogenesis of TDLs is not clearly understood. These lesions are considered to be an isolated middle type between multiple sclerosis and acute-disseminated encephalomyelitis plaques, which are the two common types of demyelinating diseases in the central nervous system $(1,8)$. Pathological features demonstrated by demyelinated diseases include hyper cellular lesions with confluent demyelination, abundant foamy macrophages containing myelin debris, reactive astrogliosis, 'relative' axonal preservation and variable perivascular and parenchymal lymphocytic inflammation. Infiltration by macrophages and lymphocytes (in particular T cells) is a main pathological feature of TDLs, which implies an association between TDLs and virus infection. A number of previous reports support the hypothesis that the pathogenesis of TDLs may be associated with infections or vaccination, similar to other demyelinating disorders (12-14). In the present study, only one case presented a history of hepatitis B vaccination and no cases were associated with infection, which may imply that TDLs is pathogenically heterogeneous.

TDLs may occur at any age groups, but are most commonly observed in adult patients in the second and third decades of their life, as observed in previous studies (12-15). It has also previously been reported that TDLs had a female prevalence similar to that of multiple sclerosis, although no gender predilection was reported $(16,17)$. However, a slight male prevalence was observed in the present study. The common presentations of TDLs mimic tumors rather than other demyelinating disorders, including increased ICP (headache and vomiting), local neurological deficits (hemiplegia and aphasia) and seizures (18). In the present study, with the exception of the TDL patients with a history of seizures, the onset of TDLs was found to be acute or sub-acute and the neurological deficits progressed faster compared with the time course for intracranial tumors, which may be as a result of the different pathological processes. The duration between onset and admission also depended on the clinical presentations. For patients with increased ICP and local neurological deficits, the duration usually ranged between days and weeks, or between months and years for patients with seizures.

For radiological analysis, MRI is generally the most sensitive imaging technique for depicting a demyelinating disease. However, TDLs present a diagnostic challenge, since they usually presents a subcortical mass in the hemisphere mimicking gliomas as a solitary lesion or metastasis as a few separate lesions $(7,8,12-14,17)$. A number of features have previously been reported to aid diagnosis, including ringlike or open-ring enhancement and central dilated veins within the lesions $(4,5,19)$. In the present study, 9 cases $(64.3 \%$; Table II) presented with ring like or open-ring enhancement. The enhancement patterns of TDLs presented in the form of an open ring with the incomplete portion of the ring on the gray matter side of the lesion, while most typical active multiple sclerosis plaques exhibited this open-ring or arc-like pattern of enhancement only in $9 \%$ of cases (20). The enhancing portion of the ring was considered to represent the leading edge of demyelination and thus favored the white matter side of the lesion. The central non-enhancing core represented a more chronic phase of the inflammatory process. In a proportion of the TDL cases in the present study, a dilated vascular structure was observed running centrally within the lesions on the T2-weighted and Gd-DTPA enhanced images; in total, 6 such cases $(42.9 \%)$ were identified in the present study. These vascular structures have been hypothesized to represent dilated veins draining towards the distended subependymal veins $(16,18,20)$. The former sign is also observed in certain cases of glioma and metastasis, while the latter sign appears to be more specific to TDLs. The pathological diagnosis may 
also be challenging simply based on the quality of the initial frozen-section specimen (4). In the present study, only 1 case was diagnosed as a TDL based on the initial frozen-section specimen result. The final diagnosis relies on the findings of immunohistopathological analysis.

Clinically, TDLs have proven to be a diagnostic dilemma for neurosurgeons. Among the spectrum of brain tumors, TDLs are misdiagnosed as gliomas, which are common neoplastic lesions. In general, gliomas with increased ICP or local neurological deficits have a longer duration between onset and admission, usually weeks to months. TDLs usually present with a more acute onset and a shorter duration compared with gliomas (21). On an MRI scan, low-grade glioma usually appears as a subcortical mass with little mass effect and surrounding edema, but with no enhancement following Gd-DTPA administration (22). However, the majority of TDLs demonstrate enhancement. High-grade gliomas present with enhancement, and with evident mass effect and surrounding edema. Therefore, cerebral isolated masses with clinical features including acute or subacute onset, neurological deficits, enhancement (particularly ring like or open-ring enhancement) with little mass effect and surrounding edema on an MRI scans should alert neurosurgeons of the possible diagnosis of TDLs.

The majority of patients with TDLs have been reported to respond favorably to corticosteroid therapy and not progress to multiple sclerosis (8). However, a number of patients presented reduced response to steroid therapy and in those cases plasma exchange was indicated (23-25). In the present study, 5 patients with biopsy received high-dose methylprednisolone therapy, demonstrating symptomatic improvement or even total relief within 1-4 weeks. On the MRI scans, all the patients demonstrated reduction or disappearance of radiographic abnormalities following steroid therapy. In patients that underwent resection, the lesions had disappeared in the neuroradiological follow-up. In the follow-up results of the current study, $\geq 90 \%$ of patients achieved a satisfactory prognosis (KPS $\geq 80$ ). However, surgical complications occurred in 3 cases: Hemiplegia in 2 patients, while 1 patient succumbed to the disease. In comparison with the confirmed positive effects and safety profile of steroid therapy, resection resulted in further unnecessary trauma in patients. Therefore, once TDLs is considered to be a possible diagnosis, biopsy or trial steroid therapy may be indicated to avoid unnecessary resection or adjunctive therapy. In the present study, no trial steroid therapy was administrated prior to surgery due to the lack of knowledge of TDLs.

A previous study has reported that TDLs are usually isolated and rarely progress to typical multiple sclerosis (9). However, a number of studies have reported that the majority of TDL patients ultimately develop clinically definite multiple sclerosis and that a minority subset developed only TDLs at last follow-up. The median time to a second clinical episode was $\sim 2-5$ years in $45-70 \%$ of patients $(9,26,27)$. In the present study, 2 patients (15.4\%) experienced recurrence and all the cases presented with the radiological features of multiple sclerosis following 2-4 years. However, the natural progression of TDLs remains uncertain. Further studies with larger sample sizes and longer follow-up are required.
In conclusion, TDLs are a rare demyelinating disorder in the central nervous system, resembling brain tumors in their clinical and radiological features and usually responding well to steroid therapy. By improving the understanding on the clinical and radiographical features of these lesions, more patients with TDLs may be correctly diagnosed prior to resection and receive reasonable treatment, which may result in an improved and satisfactory prognosis.

\section{References}

1. Love S: Demyelinating diseases. J Clin Pathol 59: 1151-1159, 2006.

2. Barkhof F, Rocca M, Francis G, et al: Validation of diagnostic magnetic resonance imaging criteria for multiple sclerosis and response to interferon beta-1a. Ann Neurol 53: 718-724, 2003.

3. Triulzi F and Scotti G: Differential diagnosis of multiple sclerosis: contribution of magnetic resonance techniques. J Neurol Neurosurg Psychiatry 64 (Suppl 1): S6-S14, 1998.

4. Masdeu JC, Quinto C, Olivera C, Tenner M, Leslie D and Visintainer P: Open-ring imaging sign: Highly specific for atypical brain demyelination. Neurology 54: 1427-1433, 2000.

5. Klawiter EC, Benzinger T, Roy A, Naismith RT, Parks BJ and Cross AH: Spinal cord ring enhancement in multiple sclerosis. Arch Neurol 67: 1395-1398, 2010.

6. Di Patre PL, Castillo V, Delavelle J, Vuillemoz S, Picard F and Landis T: 'Tumor-mimicking' multiple sclerosis. Clin Neuropathol 22: 235-239, 2003

7. Dagher AP and Smirniotopoulos J: Tumefactive demyelinating lesions. Neuroradiology 38: 560-565, 1996.

8. Lucchinetti CF, Gavrilova RH, Metz I, et al: Clinical and radiographic spectrum of pathologically confirmed tumefactive multiple sclerosis. Brain 131: 1759-1775, 2008.

9. van der Velden M, Bots GT and Endtz LJ: Cranial CT in multiple sclerosis showing a mass effect. Surg Neurol 12: 307-310, 1979.

10. Wurm G, Parsaei B, Silye R and Fellner FA: Distinct supratentorial lesions mimicking cerebral gliomas. Acta Neurochir (Wien) 146: 19-26, 2004.

11. Iwamoto K, Oka H, Utsuki S, Ozawa T and Fujii K: Late-onset multiple sclerosis mimicking brain tumor: A case report. Brain Tumor Pathol 21: 83-86, 2004.

12. Zagzag D, Miller DC, Kleinman GM, Abati A, Donnenfeld H and Budzilovich GN: Demyelinating disease versus tumor in surgical neuropathology. Clues to a correct pathological diagnosis. Am J Surg Pathol 17: 537-545, 1993.

13. Peterson K, Rosenblum MK, Powers JM, Alvord E, Walker RW and Posner JB: Effect of brain irradiation on demyelinating lesions. Neurology 43: 2105-2112, 1993.

14. Hunter SB, Ballinger WE Jr and Rubin JJ: Multiple sclerosis mimicking primary brain tumor. Arch Pathol Lab Med 111: 464-468, 1987.

15. Lucchinetti CF, Gavrilova RH, Metz I, Parisi JE, Scheithauer BW, Weigand $\mathrm{S}$, et al: Clinical and radiographic spectrum of pathologically confirmed tumefactive multiple sclerosis. Brain 131: 1759-1775, 2008.

16. Crooks V, Waller S, Smith T and Hahn TJ: The use of the Karnofsky Performance Scale in determining outcomes and risk in geriatric outpatients. J Gerontol 46: M139-M144, 1991.

17. Comi G: Mutiple sclerosis: Pseudotumoral forms. Neurol Sci 25 (Suppl 4): S374-S379, 2004.

18. Mitha AP, Scott JN, George D, Hanson A, MacRae ME and Bell RB: Tumefactive demyelinating lesions. Can J Neurol Sci 34: 362-364, 2007.

19. Given CA II, Stevens BS and Lee C: The MRI appearance of tumefactive demyelinating lesions. AJR Am J Roentgenol 182: 195-199, 2004.

20. He J, Grossman RI, Ge Y and Mannon LJ: Enhancing patterns in multiple sclerosis: evolution and persistence. AJNR Am J Neuroradiol 22: 664-669, 2001.

21. Kim DS, Na DG, Kim KH, et al: Distinguishing tumefactive demyelinating lesions from glioma or central nervous system lymphoma: Added value of unenhanced CT compared with conventional contrast-enhanced MR Imaging. Radiology 251: 467-475, 2009.

22. Enzinger C, Strasser-Fuchs S, Ropele S, et al: Tumefactive demyelinating lesions: Conventional and advanced magnetic resonance imaging. Mult Scler 11: 135-139, 2005. 
23. Cha S, Pierce S, Knopp EA, et al: Dynamic contrast-enhanced T2-weighted MR imaging of tumefactive demyelinating lesions. AJNR Am J Neuroradiol 22: 1109-1116, 2001.

24. Weinshenker BG: Therapeutic plasma exchange for acute inflammatory demyelinating syndromes of the central nervous system. J Clin Apheresis 14: 144-148, 1999.

25. Keegan M, Pineda AA, McClelland RL, Darby CH, Rodriguez M and Weinshenker BG: Plasma exchange for severe attacks of CNS demyelination: predictors of response. Neurology 58: 143-146, 2002.
26. Champs Study Group: Interferon beta-1a for optic neuritis patients at high risk for multiple sclerosis. Am J Ophthalmol 132: 463-471, 2001.

27. Confavreux C and Vukusic S: Natural history of multiple sclerosis: implications for counselling and therapy. Curr Opin Neurol 15: 257-266, 2002. 